\title{
Characteristic Analysis of Flying Process of Dispensed Object with Deceleration Parachute
}

\author{
Tao Ru-Yi, Wang Shuai, Wang Shan-Shan, and Sun Ji-Bing
}

\begin{abstract}
Characteristic of dispensed object with deceleration parachute is analyzed as it flies based on dynamic model of the object. Its flight path, velocity and accelerator of dispensed object are studied and try to find the factor to affect flies distance according to the compute result. It is found that the dynamic model is reasonable because its compute result coincides with the experiment result. Based on the dynamic model and the simulation program, both altitude of dispensing and parachute-opening and dispensing velocity can be set in advance in order to meet the demand of experiment or application. An available analysis method is supplied to improve dispensing performance of object carried by flies.
\end{abstract}

Index Terms-Air-drop, deceleration parachute, flies path, simulation.

\section{INTRODUCTION}

Since deceleration and flies steadily of dispensed object is controlled by deceleration parachute, it is important to study the flight path and attitude of dispensed object with deceleration parachute that dynamic characteristic of the system constructed by parachute and dispensed object. And it is necessary to study movement rule in the process of aerating to parachute and opening the parachute. Because its volume and frontal resistance is about ten times after aeration and opening compared with them before aeration and opening, deceleration parachute has two main functions, one is to deceleration and another is to make flying object steady [1].

The opening process of parachute for parachutist is divide four phrase by Tang jianhua [2], first is from a parachutist departing from airplane to unpack parachute, second is from the guide parachute thrown to thread flared out completely, third is from thread flared out to parachute charged, the last is charge completed.Base on the process that object and parachute are thrown and parachute opened, the process is also divided four phrases including dropping phrase, flaring out phrase, charging phrase and moving steadily phrase.

\section{WORKING PRINCIPLE}

Firstly, dispensed object and parachute are dispensed, they move under gravity and resistance. While they arrive at a certain altitude, fuze trigged at premeditation altitude, is trigged and pilot parachute is charged, the main parachute is

Manuscript received October 9, 2012; revised December 5, 2012

The authors are with the School of Energy and Power Engineering, Nanjing University of Science and Technology, Nanjing, CO210094, Jiangsu, China. (e-mail: taotaory@tom.com, 32111258@qq.com, 176050484@qq.com,Sjb_2011@tom.com). flared out. Then pilot parachute depart from the system, main parachute starts to charge and resistance increase rapidly. Finally, dispensed object slow down and move in a stead status under resistance. Fig. 1. shows the process.

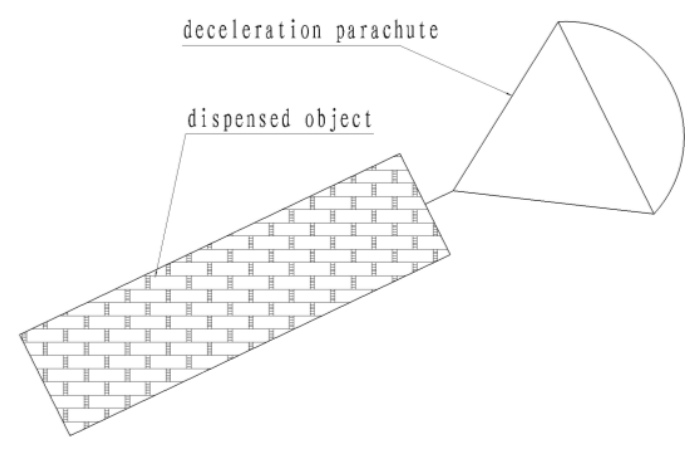

Fig. 1. Flying process sketch

\section{THEORY MODEL}

It is very complex that the move rule of system constructed by dispensed object and parachute because it is affected by shape, mass and relative position of component. There is no model can be used to describe its move process accurately. Experiment data and formula gotten from experience are applied to calculation the move path. Since main attention of the study focus on move path and pressure during the object and parachute dropping, mass model is applied to simulate the process.

As mention in Section I, the dropping process is divided four phrases and the previous phrase supply the initial condition for the next one.

\section{A. Essential Supposition}

1) Attack angle of dispensed object is zero during dropping,

2) No wind and no rain,

3) Ground surface is plane and orientation of acceleration of gravity is vertical to ground surface,

4) Coriolis force is ignored.

\section{B. Review Stage}

Formula of deceleration parachute

$$
\frac{\mathrm{d}}{\mathrm{d} t}\left[\left(m_{p}+m_{f}\right) v\right]=F_{k}-R_{p}+m_{p} g \cos \psi_{2}^{\prime} \sin \theta_{1}^{\prime}
$$

There $R_{p}=\frac{1}{2} \rho v^{2} C_{A}, C_{A}$ is eigenvector of resistance to dispensed object and parachute, $F_{k}$ is payload of opening 
parachute. $m_{f}$ is additional mass.

Payload of opening parachute is calculated

$F_{k}=R_{p}+v \frac{\mathrm{d} m_{f}}{\mathrm{~d} t}+\left(m_{p s}+m_{f}\right) \frac{\mathrm{d} v}{\mathrm{~d} t}-m_{p s} g \cos \psi_{2} \sin \theta_{1}$

For the parachute package, the resistance characteristic is expressed

$$
C_{A}= \begin{cases}K s & s_{0} \leq s \leq s_{1} \\ C_{A_{1}}+\beta\left(s-s_{1}\right)^{4} & s_{1} \leq s \leq s_{m}\end{cases}
$$

For the parachute with roundness shape and coadunate stuff, $\quad C_{A_{1}}=0.04 C_{A_{f}} \quad\left(C_{A_{f}} \quad\right.$ is resistance eigenvector)

$$
s_{1}=\lambda D_{p}
$$

$s_{m}=\alpha D_{p}, K=C_{A_{1}} / s_{1}, \beta=\left(C_{A_{f}}-C_{A_{1}}\right) /\left(s_{m}-s_{1}\right)^{4}($

$D_{p}$ is diameter of parachute) $\lambda=1.74, \alpha=6 \sim 7$.

Additional mass $m_{f}$ is [4]

$$
m_{f}=K_{f} \rho C_{A}^{3 / 2}
$$

$K_{f}$ is coefficient of additional mass, For the parachute with roundness shape and coadunate stuff, $K_{f} \approx 0.41$.

Movement formula is

$$
\left\{\begin{array}{l}
\frac{\mathrm{d} x}{\mathrm{~d} t}=v \cos \psi_{2} \cos \theta_{1} \\
\frac{\mathrm{d} y}{\mathrm{~d} t}=v \cos \psi_{2} \sin \theta_{1} \\
\frac{\mathrm{d} z}{\mathrm{~d} t}=v \sin \psi_{2} \\
\frac{\mathrm{d} \theta_{1}}{\mathrm{~d} t}=\frac{g \cos \theta_{1}}{v \cos \psi_{2}^{\prime}} \\
\frac{\mathrm{d} v}{\mathrm{~d} t}=g \cos \psi_{2} \sin \theta_{1}-\frac{F_{k}-R_{1}}{m_{1}} \\
F_{k}=R_{p}+v \frac{\mathrm{d} m_{f}}{\mathrm{~d} t}+\left(m_{p s}+m_{f}\right) \frac{\mathrm{d} v}{\mathrm{~d} t}-m_{p s} g \cos \psi_{2} \sin \theta_{1} \\
\frac{\mathrm{d} \psi_{2}}{\mathrm{~d} t}=-\frac{g \sin \psi_{2} \sin \theta_{1}}{v} \\
\frac{\mathrm{d} m_{f}}{\mathrm{~d} t}=\frac{3}{2} K_{f} \rho C_{A}^{1 / 2} \frac{\mathrm{d} C_{A}}{\mathrm{~d} s} \\
m_{f}=K_{f} \rho C_{A}^{3 / 2} \\
C_{A}= \begin{cases}K s & s_{0} \leq s \leq s_{1} \\
C_{A_{1}}+\beta\left(s-s_{1}\right)^{4} & s_{1} \leq s \leq s_{m}\end{cases} \\
\frac{\mathrm{d} C_{A}}{\mathrm{~d} s}= \begin{cases}K & s_{0} \leq s \leq s_{1} \\
4 \beta\left(s-s_{1}\right)^{3}\end{cases}
\end{array}\right.
$$

$R_{1}$ is air resistance.

\section{UNITS}

Parameter is used to simulate the process of a certain dispensed object and parachute.

Parameter of dispensing (Mass of dispensed object is $340.0 \mathrm{~kg}$, Length of dispensed object is $1.783 \mathrm{~m}$, and Diameter dispensed object is $0.430 \mathrm{~m}$ )

Parameter of deceleration parachute(Mass of parachute is $15.0 \mathrm{Kg}$, Whole length of parachute is $3.0 \mathrm{~m}$,Parachute rope is $1.5 \mathrm{~m}$, Radius of opening parachute is $1.2 \mathrm{~m}$ )

Dispensing condition:

1) Dispensing state $A$ :

Dispensing attitude is $800 \mathrm{~m}$, dispensing velocity is $800 \mathrm{~km} / \mathrm{h}$, parachute is opened while attitude is $300 \mathrm{~m}$.

2) Dispensing state $B$ :

Dispensing attitude is $500 \mathrm{~m}$, dispensing velocity is $500 \mathrm{~km} / \mathrm{h}$, parachute is opened while attitude is $200 \mathrm{~m}$.

Base on the calculation model, simulation result is shown in Fig. 2-Fig. 8.

As shown in Fig. 2 and Fig. 3, dip angle of movement path of dispensed object change slowly both at state B and at state A in the first phrase. Resistance of dispensed object and parachute increase and obliquity of movement path change faster than phrase 1 after deceleration parachute is opened. Obliquity of movement path almost is a right angle during the object is near to ground.

Fig. 4 shows that the flying distance of dispensed object is increasing with the altitude and velocity when it is dispensed.

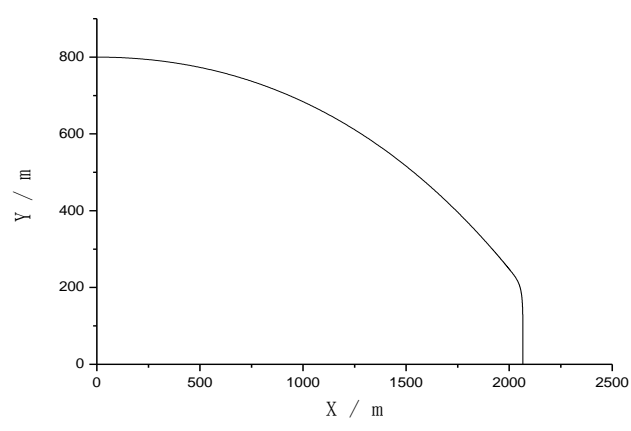

Fig. 2. Carve of movement path at state A

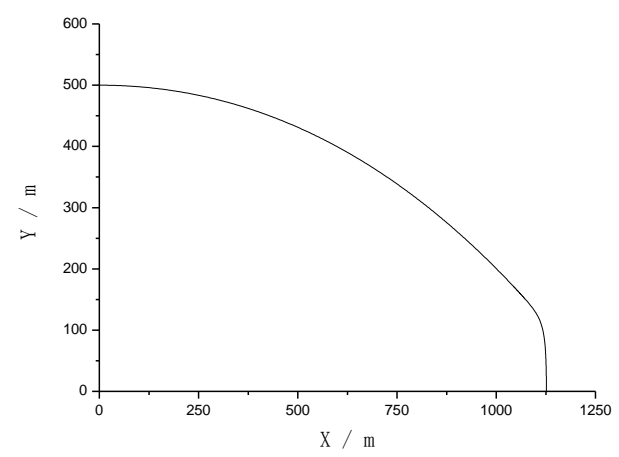

Fig. 3. Carve of movement path at state B

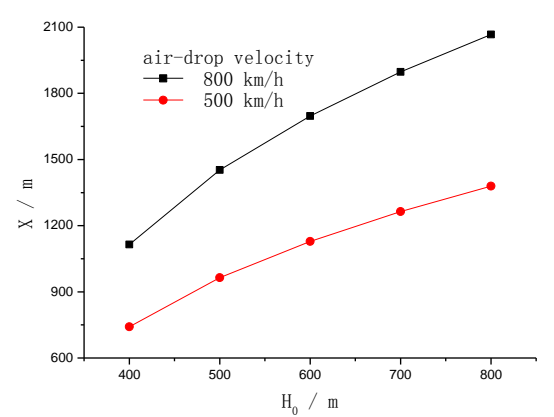

Fig. 4. Journey carve at different altitude and velocity of dispensing 
It is necessary to study the change of acceleration and velocity during object flying, because it is a main factor to decide if object can be dispensed triumphantly. It is shown that both velocity and acceleration change observably during the dispensing process in the Fig. 5, Fig. 6, Fig. 7, and Fig. 8. Velocity of system change slowly in the phrase 1 , and it change fast in the phrase 2 that the deceleration parachute is charged., it need about 2 or 3 second that the velocity change slowly again. The velocity change is study at state A, state B and other state, the result shows that the velocity is about $16.5 \mathrm{~m} / \mathrm{s}$ when the dispensed object move steadily no mater the difference of dispensing altitude and velocity. So a conclusion is reached that velocity of dispensed object in phrase 4 is decided by the attribute of system constructed with dispensed object and deceleration parachute and not be affected by the dispensing altitude and dispensing velocity.

Acceleration of the system changes obviously during the process, the absolute value of acceleration is increasing with parachute is charged in phrase 2, and its maximal value occurs while the parachute is charged fully since the resistance is max at this time. Acceleration becomes less gradually after phrase 2 until become zero and move steadily.

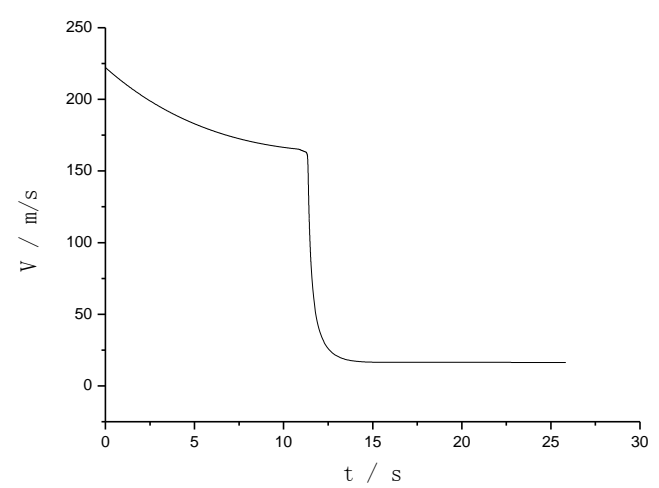

Fig. 5. Carve of velocity at state A

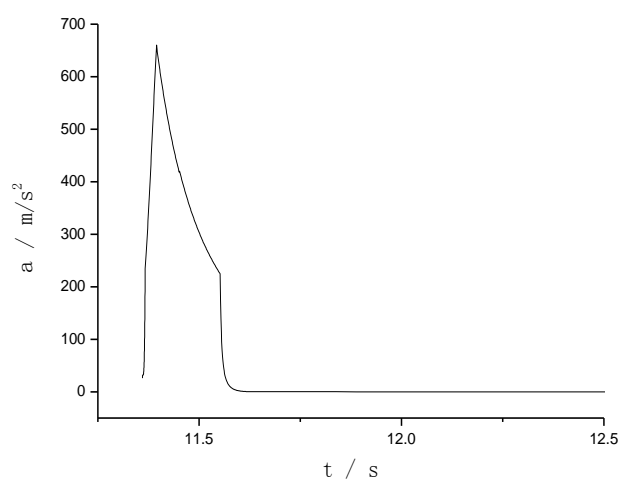

Fig. 6. Carve of absolute value of acceleration at state A

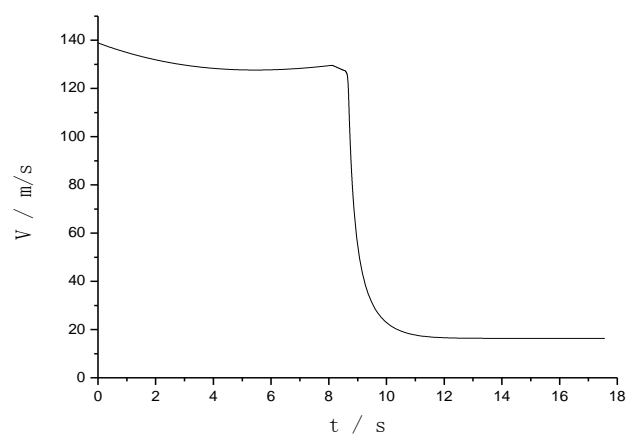

Fig. 7. Carve of velocity at state B

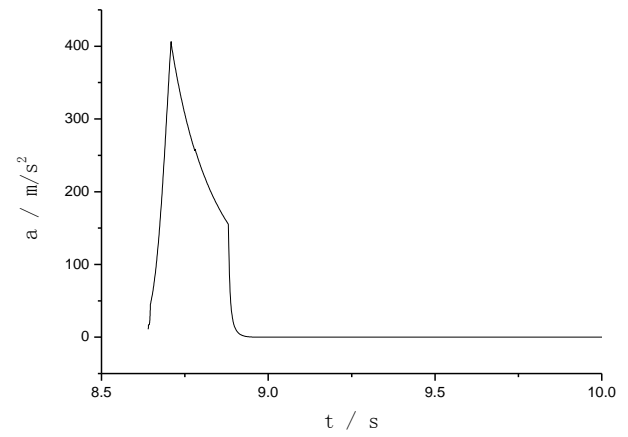

Fig. 8. Carve of absolute value of acceleration at state B

\section{CONCLUSION}

Base on the characteristic of dispensed object with deceleration parachute during flying, dynamic model is constructed and simulation program is developed to get simulation result. Then movement path, velocity and acceleration rules of dispensed object are studied and effect of dispensing altitude and velocity to flying distance is analysed. By the simulation program, not only the object position, velocity, obliquity and time can be calculated but also to designed dispensing altitude, dispensing velocity and opening parachute altitude in order to meet the application. It supplied a method to improve the performance to dispense object with deceleration parachute.

\section{REFERENCES}

[1] L.-R. Wang, Theory and Application of Parachute, Beijing: public of space navigation, 1997.

[2] J.-H. Tang, Mathematical model and numerical arithmetic of opening parachute process, M.S. thesis, Nanjing University of aeronautics and astronautics, 1988, pp. 7-12.

[3] W.-X. Gong, "Added mass and parachute opening dynamic load of parachute," Acta aeronautica et astronautica sinica, vol. 16, no. 1, pp. 84-86, January 1995.

[4] A J. Michael, Experimental Investigation of Added Mass during Parachute Deceleration Preliminary Result, AIAA 29120856, 1991.

[5] S.-L. Zhao, C Programming, Beijing, Higher Education Press, China, 2010, vol. 9, pp. 190-226.

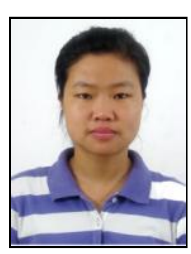

Tao Ru-Yi was born in 1980. Now she is working as a lecturer on numerical calculation in Nanjing University of Science and Technology. 